\title{
Components of Panax notoginseng saponins enhance the cytotoxicity of cisplatin via their effects on gap junctions
}

\author{
CUILING ZHANG ${ }^{1}$, XUHUI TONG ${ }^{1}$, BENQUAN $^{2}{ }^{2}$, XIAOBING YU $^{3}$, \\ SHUYING DONG ${ }^{1}$, SUZHI ZHANG ${ }^{3}$, XIAOMING LI ${ }^{1}$ and MEILING YU ${ }^{1,2}$ \\ ${ }^{1}$ Department of Pharmacology, Bengbu Medical College, Bengbu, Anhui 233030; ${ }^{2}$ Department of Pharmacy, \\ The First Affiliated Hospital of Bengbu Medical College, Bengbu, Anhui 233004; ${ }^{3}$ Department of Pharmacology, \\ Zhongshan School of Medicine, Sun Yat-Sen University, Guangzhou, Guangdong 510080, P.R. China
}

Received January 26, 2013; Accepted July 12, 2013

DOI: $10.3892 / \mathrm{mmr} .2013 .1597$

\begin{abstract}
Previously, Panax notoginseng saponin (PNS)-induced enhancement of gap junction (GJ) formation or function was observed to be responsible for the increased cytotoxic action of cisplatin. PNS has three constituents, ginsenoside $\mathrm{Rg} 1$ and $\mathrm{Rb}$, and notoginsenoside $\mathrm{R} 1$. The active compounds in PNS responsible for enhancing the cytotoxicity of cisplatin remain unknown. Thus, the effects of the main components of PNS on the cytotoxicity of cisplatin were investigated, as well as the correlation with the modulation of GJ function in transfected HeLa cells. The cytotoxicity of cisplatin $(0.25-1 \mu \mathrm{g} / \mathrm{ml})$ was increased in the presence of GJs. By contrast, the cytotoxicity of cisplatin was decreased when GJs were inhibited by a GJ blocker or by the inhibition of connexin expression. Ginsenoside $\operatorname{Rg} 1(100 \mu \mathrm{M})$ and notoginsenoside $\mathrm{R} 1(100 \mu \mathrm{M})$ were observed to significantly enhance cisplatin cytotoxicity in cells with functional GJs. Ginsenoside Rb1 had no effect on the cytotoxicity of cisplatin in the presence or absence of functional GJs. Cell exposure to ginsenoside Rg1 and notoginsenoside R1 for $4 \mathrm{~h}$ led to significant enhancement of a dye-coupled GJ in a dose-dependent manner; however, no effect was observed in cells exposed to ginsenoside Rb1. The present results indicate that ginsenoside $\operatorname{Rg} 1$ and notoginsenoside $\mathrm{R} 1$ are the active compounds responsible for enhancing the cytotoxic action of cisplatin induced by PNS in the presence of functional GJs.
\end{abstract}

\section{Introduction}

Combination chemotherapy is an effective treatment for cancer and is often more effective than single chemotherapy due to additive or synergistic effects. In addition, adjuvant therapy decreases dose-related toxicities. Therefore, identi-

Correspondence to: Dr Meiling Yu, Department of Pharmacy, The First Affiliated Hospital of Bengbu Medical College, 287 Changhuai Road, Bengbu, Anhui 233004, P.R. China

E-mail: yumeiling409@sohu.com

Key words: ginsenoside Rg1, ginsenoside Rb1, notoginsenoside R1, cisplatin, gap junction fying nontoxic chemoadjuvants, including natural compounds, may be an essential step in advancing cancer treatment. Panax notoginseng saponins (PNSs) are extracted from the perennial herb, notoginseng. The main components are ginsenoside Rg1 and Rb1, and notoginsenoside R1. Previous studies have indicated that PNS and its components may enhance the cytotoxicities of a number of chemotherapy agents (1-3).

Gap junctions (GJs) are specialized cell-cell junctions that directly link the cytoplasm of neighboring cells in the majority of vertebrate organs. A number of specific functions, including homeostasis maintenance, morphogenesis, cell differentiation and growth control in multicellular organisms, have been associated with GJs.

Previous studies have shown that GJs promote apoptosis induced by specific chemical agents in normal or tumor cells $(4,5)$. A study by Wang et al confirmed that enhancing the cytotoxicities of cisplatin and etoposide depends on GJ intercellular communication (GJIC) (6). A hypothesis derived from these studies is that a molecular 'death signal' caused by the induction of apoptotic or necrotic processes in one cell may be transmitted to neighboring cells via GJs. Thus, a number of studies have shown that an increase in the intercellular spread of the 'death signal' caused by the enhancement of GJ formation or function may contribute to increased cytotoxic action of cisplatin (4-8). By contrast, blocking GJ-signaling decreases the intercellular cytotoxicity of cisplatin $(8,9)$.

Our previous study showed that PNS increases the cytotoxicity of cisplatin by enhancing GJ formation or function (7); however, the mechanism of action remains poorly defined. In the current study, the effects of the main constituents of PNS, ginsenoside Rg1 and Rb1, and notoginsenoside R1, on the cytotoxicity of cisplatin, were investigated, as well as the correlation between the effects and modulation of GJ function in transfected HeLa cells. The active compounds in PNS responsible for the enhancement of the cytotoxic action of cisplatin were investigated and the cellular mechanisms underlying this action were identified.

\section{Materials and methods}

Materials. Ginsenoside Rg1 and Rb1, and notoginsenoside R1 were purchased from Yunnan Kingpanax (Group) Co., Ltd. 
(Kunming, Yunnan, China). Cisplatin, anti-hemagglutinin (HA) mouse IgG, anti- $\beta$-actin and dimethylsulfoxide (DMSO) were purchased from Sigma-Aldrich (St. Louis, MO, USA). Hygromycin B, G418, 2-aminoethoxydiphenyl borate (2-APB) and doxycycline were purchased from Calbiochem (La Jolla, CA, USA). Cell culture reagents, calcein-acetoxymethyl ester (calcein-AM) and TRIzol were obtained from Invitrogen Life Technologies (Carlsbad, CA, USA). Secondary antibodies for western blotting were obtained from Amersham Pharmacia Biotech (Piscataway, NJ, USA). All other reagents were from Sigma-Aldrich unless stated otherwise.

Cell lines and cell culture. Cell lines expressing heteromeric connexin32/connexin26 (Cx32/Cx26) were cultured as described previously (10). In this cell line, a single bidirectional tetracycline-inducible promoter controls the expression of the two connexins. The $\mathrm{Cx} 26$ has a thrombin-cleavable $\mathrm{C}$-terminal epitope tag (3.2 kDa) which includes an HA epitope.

Transfected HeLa cells were grown at $37^{\circ} \mathrm{C}$ in Dulbecco's modified Eagle's medium supplemented with $10 \%$ fetal bovine serum, $100 \mu \mathrm{g} / \mathrm{ml} \mathrm{G} 418$ sulfate and $200 \mu \mathrm{g} / \mathrm{ml}$ hygromycin B. Connexin expression was induced with $1 \mu \mathrm{g} / \mathrm{ml}$ doxycycline for $48 \mathrm{~h}$ prior to experimental treatments.

'Parachute' dye-coupling assay. The assay for GJ function was performed as described previously $(10,11)$. Briefly, donor and receiver cells were grown to $70-80 \%$ confluency in 12 -well plates. Donor cells from one well were incubated in growth medium supplemented with a freshly prepared solution of $5 \mu \mathrm{M}$ calcein-AM for $30 \mathrm{~min}$ at $37^{\circ} \mathrm{C}$. Calcein-AM is intracellularly converted into the GJ-permeable dye, calcein. Subsequently, the donor cells were trypsinized and seeded onto the receiver cells at a ratio of 1:150. Cells were allowed to adhere to the monolayer of receiver cells and form GJs for $4 \mathrm{~h}$ at $37^{\circ} \mathrm{C}$ and then examined by fluorescence microscopy. The average number of receiver cells containing calcein/donor cell was considered as a measure of the degree of GJIC.

Western blotting. Western blotting was performed as previously described (7). Mouse anti-HA clone HA-7 IgG was used at a 1:1,000 dilution and the secondary antibody was used at a 1:2,000 dilution. Anti- $\beta$-actin and the secondary antibody were used at a 1:8,000 dilution. All western blotting exposures were in the linear range of detection and the intensities of the resulting bands were quantified using the Quantity One software on a GS-800 densitometer (Bio-Rad, Hercules, CA, USA).

Standard colony-forming assay. All exposures to cisplatin and the components of PNS, i.e. ginsenoside Rg1 and Rb1, and notoginsenoside R1, were performed for 1 or $4 \mathrm{~h}$, respectively, in the dark. When combined with cisplatin, the components of PNS were added to cells $3 \mathrm{~h}$ prior to cisplatin. 2-APB, dissolved in DMSO at $2.3 \mathrm{mg} / \mathrm{ml}$ and diluted to a final concentration of $2.3 \mu \mathrm{g} / \mathrm{ml}$ in culture medium, was added to the cells $1 \mathrm{~h}$ prior to cisplatin.

Cisplatin cytotoxicity was assessed as previously described (12). This assay is a standard colony-forming assay, adapted for use at high and low cell density, according to conditions in which junctional channel formation is permitted or not permitted, respectively. For high density conditions, cells were seeded at 30,000 cells $/ \mathrm{ml}$ to obtain $70-100 \%$ confluence when exposed to cisplatin. At this density, there was substantial opportunity for GJ formation since each cell was in contact with an average of 3-5 other cells. For low-density conditions, cells were seeded at 100 cells/ml into 6-well plates. Following 4-h treatment, cells were exposed to cisplatin, washed with PBS and replenished with fresh media. At low seeding density, GJ formation was prevented as cells did not contact each other. Cells were rinsed and assessed for colony formation. Colony formation was normalized to the colony forming efficiency of nondrug-treated cells using the following formula: Surviving fraction $=$ drug-treated cell clone number/nondrug-treated cell clone number.

To avoid discrepancies in results caused by cells being in different stages of the cell cycle, serum-free medium was used for $24 \mathrm{~h}$ prior to exposure of cisplatin to maintain cells synchronously in the G1 phase. There was no significant difference in plating efficiency between the low- and high-density cultures in the untreated samples (data not shown).

$R N A$ extraction and reverse transcription polymerase chain reaction $(R T-P C R)$. TRIzol reagent was used to extract total RNA according to the manufacturer's instructions. Complementary DNA (cDNA) was synthesized from $1 \mu \mathrm{g}$ RNA using the standard procedure with avian myeloblastosis virus reverse transcriptase (Promega Corporation, Madison, WI, USA). For PCR quantification, $2 \mu 1$ cDNA was amplified in a $20 \mu \mathrm{l}$ standard PCR. PCR was performed by initial denaturation at $94^{\circ} \mathrm{C}$ for $3 \mathrm{~min}, 36$ cycles of $94^{\circ} \mathrm{C}$ for $45 \mathrm{sec}$, $55^{\circ} \mathrm{C}$ for $45 \mathrm{sec}$ and $72^{\circ} \mathrm{C}$ for $45 \mathrm{sec}$, and a final extension for $10 \mathrm{~min}$ at $72^{\circ} \mathrm{C}$, followed by termination at $4^{\circ} \mathrm{C}$. RT-PCR was performed using the following pairs of primers (Invitrogen Life Technologies) for the semiquantitative assessment: rat Cx26 forward, 5'-TCTCTCACATCCGGCTCTGG-3' and reverse, 5'-TCCGTTTCTTTTCGTGTCTCC-3', yielding a 102 bp product; and human $\beta$-actin forward, 5'-CGTGGACATCCGCAAAGAC-3' and reverse, 5'-GCATTTGCGGTGGACGAT-3', yielding a 256-bp product. The detection of $\beta$-actin transcripts provided an internal control in PCR, standardizing the quantity of input cDNA. PCR products were separated by electrophoresis on a $1.5 \%$ agarose gel and visualized under UV using the gel documentation system (Bio-Rad).

Statistical analysis. Statistical analyses between groups were performed using an unpaired Student's t-test with SigmaPlot 10.0 software (Jandel Scientific, San Rafael, CA, USA). P<0.05 was considered to indicate a statistically significant difference.

\section{Results}

Differential effects of cell density on cytotoxicity of cisplatin. Since the formation of GJ channels relies on the end-to-end docking of two hemichannels in adjacent cells, GJIC may occur when cells contact each other. At low density, cells were seeded at 100 cells/ml into 6-well plates. These cells were not in direct contact with each other and had no opportunities to form GJs. However, at high density, when cells were seeded at 30,000 cells $/ \mathrm{ml}$, GJ formation was possible since the cells 
were in contact with one another. To determine the effect of short-term cisplatin exposure on the survival of HeLa cells expressing Cx32/Cx26, colony formation was performed in the two culture conditions. As shown in Fig. 1, the clonogenic survival of cells at low and high densities was reduced by treatment with cisplatin $(0.25-1 \mu \mathrm{g} / \mathrm{ml})$ for $1 \mathrm{~h}$. Compared with the samples at low density, the toxic effect of cisplatin was substantially greater in the samples at high density, indicating that the cytotoxicity of cisplatin is cell density dependent. The concentration of cisplatin used was within the therapeutic range reached in tissue during chemotherapy (13).

Density dependence of cisplatin response is mediated by GJIC. From the aforementioned results, cell density was observed to contribute to the cytotoxicity of cisplatin and GJ formation was noted to depend on cell density. Thus, GJIC was hypothesized to mediate the density dependence of the cisplatin response. To investigate the role of GJIC in cisplatin sensitivity, GJ function was examined using two methods: the doxycycline induction of connexin expression and the pharmacological inhibition of junctional channels by 2-APB (14). HeLa cells transfected with $\mathrm{Cx} 32 / \mathrm{Cx} 26$ were used in the current study and connexin expression was induced with $1 \mu \mathrm{g} / \mathrm{ml}$ doxycycline for $48 \mathrm{~h}$. The results of western blotting and 'parachute' dye-coupling assay showed that the expression of $\mathrm{Cx} 32 / \mathrm{Cx} 26$ and dye-coupling were significantly induced by doxycycline (Fig. 2A and B). In low cell density cultures, without GJIC, there was no significant difference in cisplatin survival between the doxycycline-induced and uninduced cells, with and without connexin expression, respectively, (Fig. 2C). However, cells treated with doxycycline were more sensitive to cisplatin compared with untreated cells at high density (Fig. 2C). Incubating the cells with 2 -APB $(2.3 \mu \mathrm{g} / \mathrm{ml})$, a membrane-permeable reagent that was verified to inhibit dye-coupling in HeLa cells (Fig. 2B) substantially increased cell survival in high-density cells, with GJIC (Fig. 2D). However, at low cell density, 2-APB had no effect on cisplatin toxicity (Fig. 2D). The 2-APB-mediated reduction of GJIC and the doxycycline-induced enhancement of GJ function affected the cytotoxicity of cisplatin at high cell density, which is in agreement with the hypothesis that GJIC mediates the cytotoxicity of cisplatin at high cell densities.

Effects of ginsenoside Rgl and Rbl, and notoginsenoside RI on the cytotoxicity of cisplatin. Our previous study showed that PNS enhanced the function of GJ and the cytotoxicity of cisplatin in high-density cultures, with GJ formation (7). Ginsenoside Rg1 and Rb1, and notoginsenoside R1 were shown to be essential effectors. To assess the role of these components in modulating the cytotoxicity of cisplatin, the effects of ginsenoside Rg1 and Rb1, and notoginsenoside R1 on cisplatin-induced cytotoxicity in HeLa cells were examined.

Cells seeded at high or low cell densities were treated with ginsenoside $\mathrm{Rg} 1$ and Rb1, and notoginsenoside R1 for $3 \mathrm{~h}$, followed by exposure to $0.5 \mu \mathrm{g} / \mathrm{ml}$ cisplatin and these components for $1 \mathrm{~h}$. The clonogenic survival of HeLa cells was examined 7 days following exposure to cisplatin and ginsenoside Rg1 and Rb1, and notoginsenoside R1. Ginsenoside Rg1 and notoginsenoside R1 had no effect on cisplatin toxicity in low-density cultures; however, the cytotoxicity of cisplatin was enhanced in high-density cultures (Fig. 3A and B). By contrast,

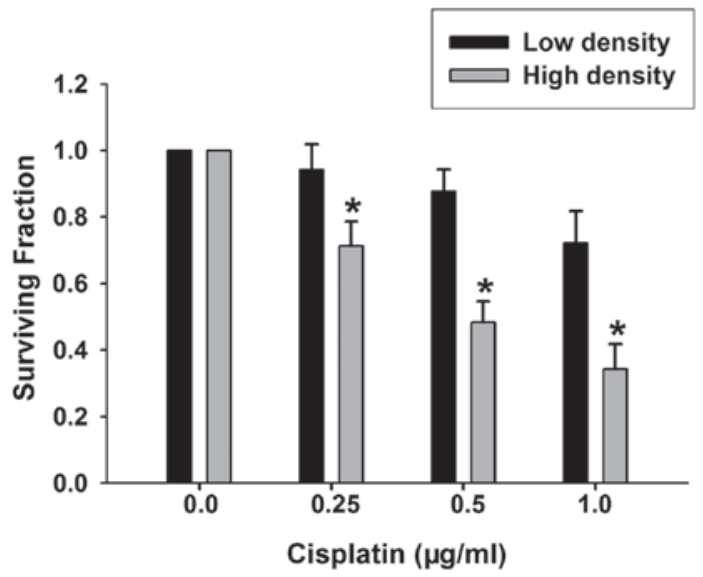

Figure 1. Effects of cell density on the cytotoxicity of cisplatin. The clonogenic survival of cells exposed to a range of cisplatin concentrations $(0.2-1 \mu \mathrm{g} / \mathrm{ml})$ for $1 \mathrm{~h}$ at low $\left(100\right.$ cells $\left./ \mathrm{cm}^{2}\right)$ or high $\left(30,000\right.$ cells $\left./ \mathrm{cm}^{2}\right)$ cell density ( $\mathrm{n}=6 ;{ }^{*} \mathrm{P}<0.05$, vs. low cell density group).

A

\section{Cx32/26t}

$\beta$-actin

B
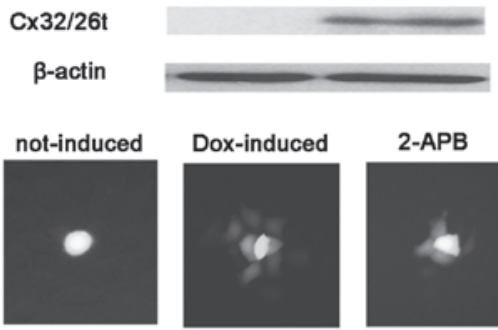

2-APB

C

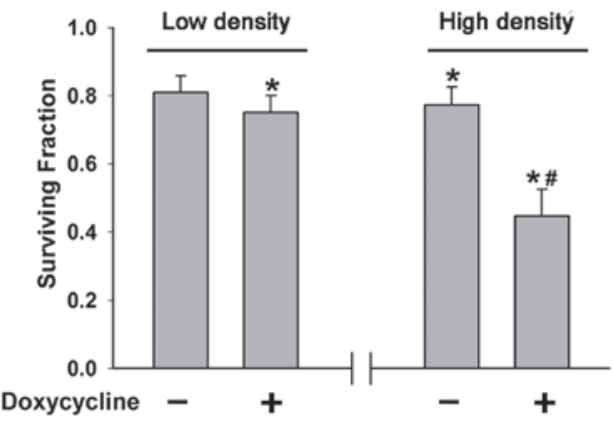

D

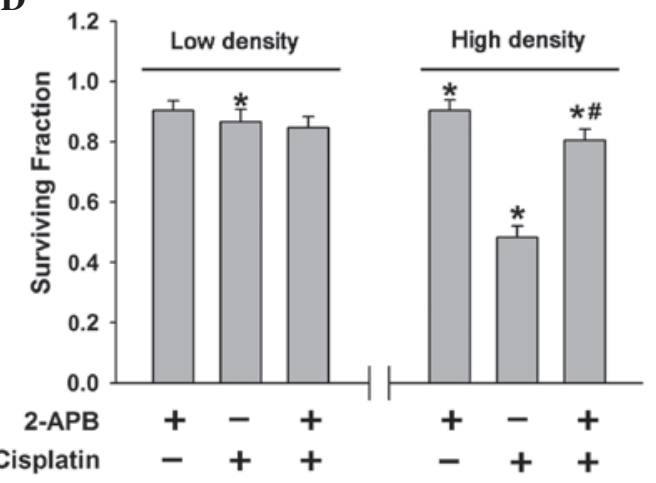

Figure 2. Density dependence of cisplatin response is mediated by GJIC (A and B) Effects of doxycycline $(1 \mu \mathrm{g} / \mathrm{ml})$ on Cx32/Cx26 expression and gap junction function in HeLa cells. (C) Cisplatin survival response in doxycycline-induced and uninduced cells $\left(\mathrm{n}=4 ;{ }^{*} \mathrm{P}<0.05\right.$, vs. control group; ${ }^{*} \mathrm{P}<0.05$, vs. not-induced group). (D) Effects of 1-h treatment of cells with 2-APB $(2.3 \mu \mathrm{g} / \mathrm{ml})$ on the cytotoxicity of cisplatin at low and high cell density $(\mathrm{n}=5$; ${ }^{\text {" }} \mathrm{P}<0.05$ vs. control group; ${ }^{~} \mathrm{P}<0.05$, vs. cisplatin group). Dox, doxycycline; 2-APB, 2-aminoethoxydiphenyl borate; GJIC, gap junction intercellular communication. 
A

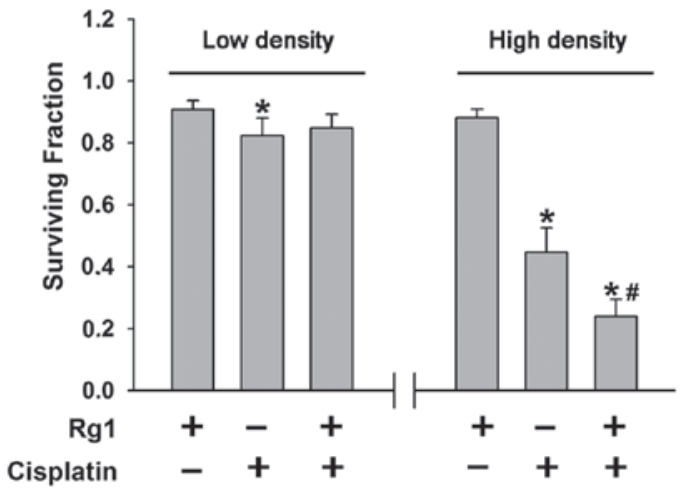

B

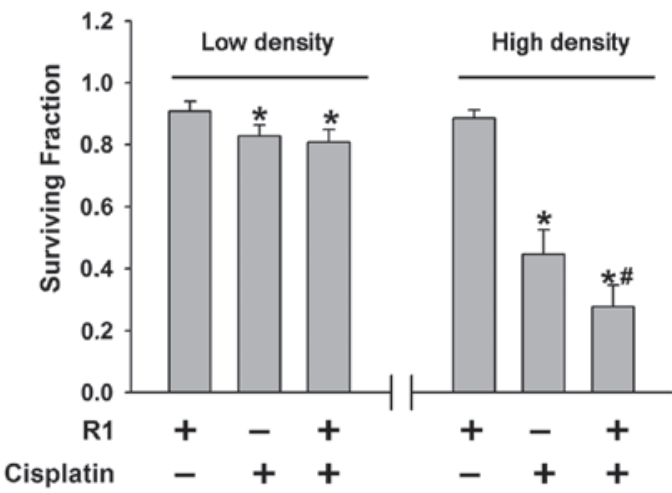

C

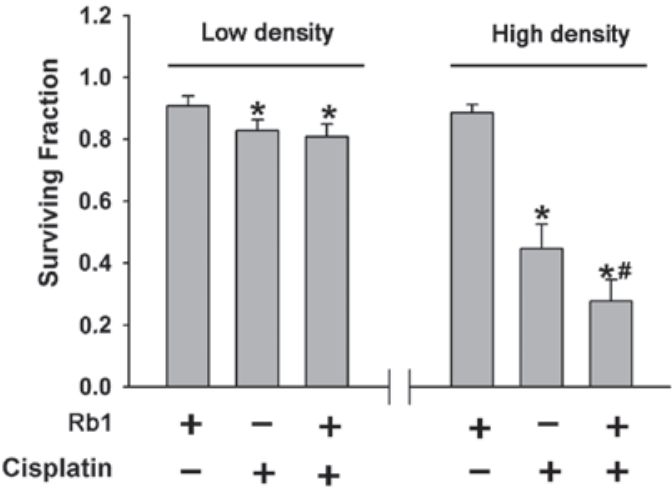

Figure 3. Effects of ginsenoside $\mathrm{Rg} 1$ and $\mathrm{Rb} 1$, and notoginsenoside $\mathrm{R} 1$ on cisplatin cytotoxicity. (A) Effects on cisplatin cytotoxicity when cells were treated with ginsenoside $\operatorname{Rg} 1(\operatorname{Rg} 1,100 \mu \mathrm{M})$, (B) notoginsenoside R1 (R1, $100 \mu \mathrm{M})$ and $(\mathrm{C})$ ginsenoside $\mathrm{Rb} 1(\mathrm{Rb} 1,100 \mu \mathrm{M})$ for $4 \mathrm{~h}$, with or without treatment with cisplatin $(0.5 \mu \mathrm{g} / \mathrm{ml})$ for $1 \mathrm{~h}$ at low and high cell densities ( $\mathrm{n}=6 ;{ }^{*} \mathrm{P}<0.05$, vs. control group; ${ }^{\#} \mathrm{P}<0.05$, vs. cisplatin group).

ginsenoside Rb1 had no effect on low- or high-density cultures (Fig. 3C). Thus, ginsenoside Rg1 and notoginsenoside R1 enhance the toxicity of cisplatin in high-density cultures where there is an opportunity for GJ formation.

Effects of ginsenoside Rg1 and Rb1, and notoginsenoside RI on GJ function. Ginsenoside Rg1 and notoginsenoside R1 affected cisplatin toxicity at high cell densities indicating that the protective effects may be mediated by GJ channels. To investigate this hypothesis, the effects of ginsenoside Rg1 and $\mathrm{Rb} 1$, and notoginsenoside R1 on dye-coupling between cultured cells were examined by 'parachute' dye-coupling assay. The results showed that ginsenoside $\operatorname{Rg} 1$ and notoginsenoside R1 markedly increased the dye spread from donor to receiver cells in a dose-dependent manner (Fig. 4A and B); however, ginsenoside $\mathrm{Rb} 1$ had no effect on dye coupling between cultured cells (Fig. 4C). Thus, ginsenoside Rb1 did not affect the cytotoxicity of cisplatin, while ginsenoside $\operatorname{Rg} 1$ and notoginsenoside R1 enhanced the toxicity of cisplatin in the high-density cultures with GJ formation. To determine whether ginsenoside Rg1 and notoginsenoside R1 affected connexin expression, the expression of $\mathrm{Cx} 32 / \mathrm{Cx} 26$ in cells induced with doxycycline, followed by exposure to ginsenoside $\mathrm{Rg} 1$ and notoginsenoside R1, was assessed by western blotting. Treatment with ginsenoside $\mathrm{Rg} 1$ and notoginsenoside R1 for $4 \mathrm{~h}$ increased $\mathrm{Cx} 32 / \mathrm{Cx} 26$ expression in a dose-dependent manner (Fig. 4D and E), indicating that the enhancement of GJIC by ginsenoside $\mathrm{Rg} 1$ and notoginsenoside R1 is primarily attributable to increased connexin expression.

Effects of ginsenoside Rgl and notoginsenoside RI on Cx32/Cx26 mRNA levels. The effects of ginsenoside Rg1 and notoginsenoside $\mathrm{R} 1$ on $\mathrm{Cx} 32 / \mathrm{C} \times 26$ mRNA levels were investigated using semi-quantitative endpoint RT-PCR. Fig. 5 shows that treating HeLa cells with ginsenoside Rg1 and notoginsenoside $\mathrm{R} 1$ for $4 \mathrm{~h}$ did not affect the levels of $\mathrm{Cx} 32 / \mathrm{Cx} 26$ mRNA expression. This result indicates that the enhancement of $\mathrm{Cx} 32 / \mathrm{Cx} 26$ expression by ginsenoside $\mathrm{Rg} 1$ and notoginsenoside R1 occurred at the post-transcriptional level.

\section{Discussion}

The current results indicate that ginsenoside $\mathrm{Rg} 1$ and notoginsenoside R1 are the active compounds responsible for enhancing the cytotoxic action of cisplatin induced by PNS. Ginsenoside Rb1 had no effect on the cytotoxicity of cisplatin in the presence or absence of functional GJs. Our previous study demonstrated that the enhancement of cisplatin cytotoxicity by PNS is mediated by upregulating GJ function in HeLa cells (7). Treatment with PNS for $4 \mathrm{~h}$ increased dye coupling via $\mathrm{C} \times 32 / \mathrm{C} \times 26$ channels. The present study showed that pre-treatment of cells with ginsenoside $\mathrm{Rg} 1$ and notoginsenoside R1 for $4 \mathrm{~h}$ markedly increased the dye spread from donor to receiver cells in a dose-dependent manner; however, ginsenoside Rb1 had no effect on dye coupling. Ginsenoside $\mathrm{Rg} 1$ and notoginsenoside $\mathrm{R} 1$ also increased Cx32/Cx26 expression; however, Cx32/Cx 26 mRNA levels were not affected. Our previous study confirmed that the long-term treatment of HeLa cells with cisplatin results in a reduction of Cx32/Cx26 expression and that the administration of PNS reversed this reduction. These results indicate that enhancement of Cx32/Cx 26 expression by PNS, ginsenoside Rg1 and notoginsenoside $\mathrm{R} 1$ are due to alterations in the stability of the protein, i.e. inhibition of $\mathrm{Cx} 32 / \mathrm{Cx} 26$ degradation and/or modulation of translation.

Previous studies have demonstrated that cisplatin toxicity is enhanced by the presence of functional GJs $(7,9,12)$. GJ expression allows cisplatin to promote apoptosis, cell cycle arrest and the downregulation of BCL-2 in bladder cancer cell lines (15). An inference derived from these studies is that a 'death signal' induced by the apoptotic or necrotic processes of one cell may be transmitted to neighboring cells via GJs. In the present study, all cells were exposed to the same dose of drug to mimic drug administration in vivo. In theory, the presence of GJIC should 
A

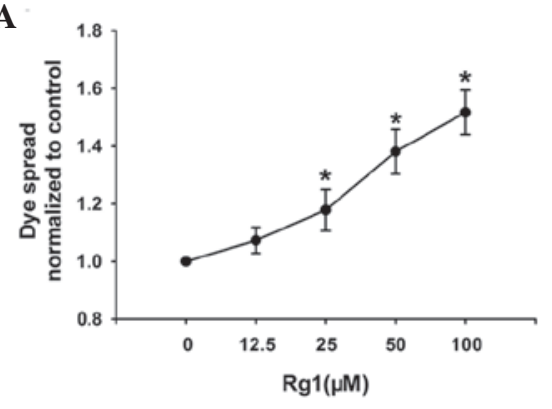

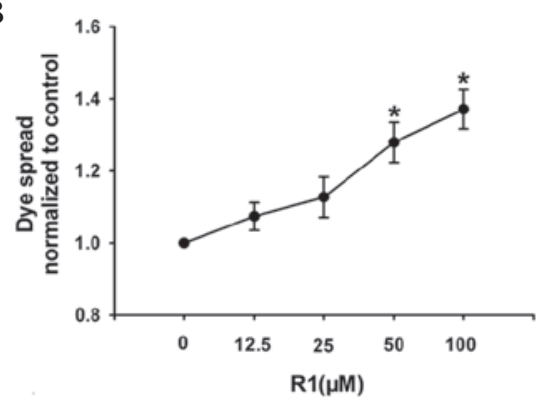

C

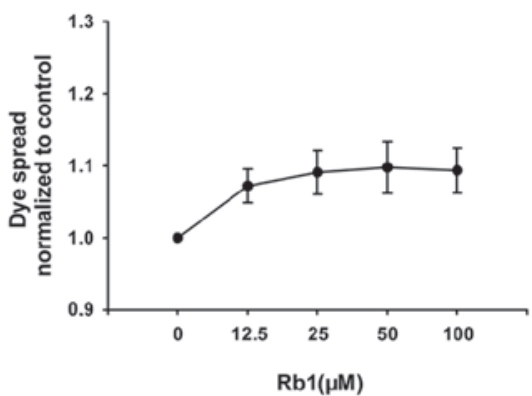

D

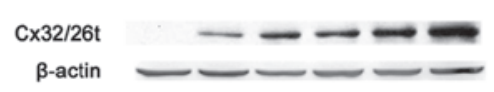

$\mathbf{E}$
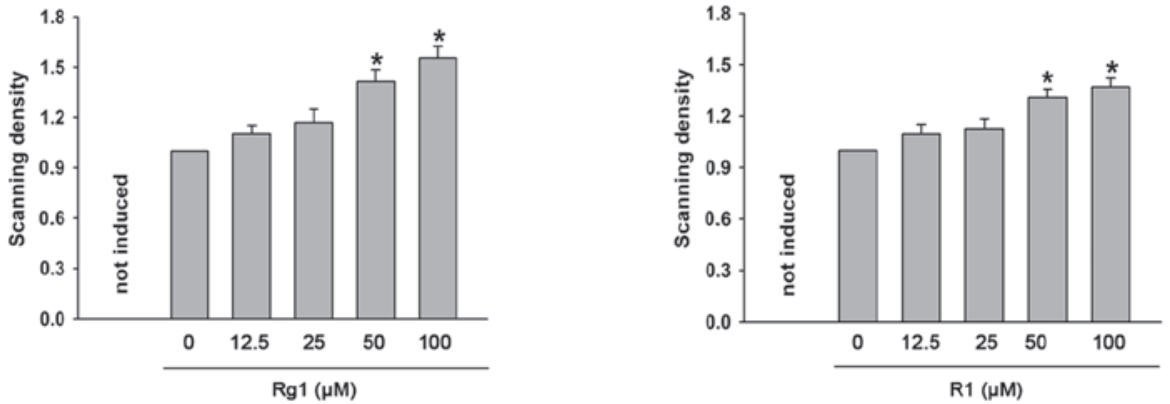

Figure 4. Effects of ginsenoside Rg1 and Rb1, and notoginsenoside R1 on dye-coupling through GJs composed of Cx32/Cx26 in HeLa cells. (A) The effects of 4-h cell treatment with ginsenoside $\operatorname{Rg} 1(\mathrm{Rg} 1,12.5-100 \mu \mathrm{M})$, (B) notoginsenoside $\mathrm{R} 1$ (R1, 12.5-100 $\mu \mathrm{M}$ ) or (C) ginsenoside Rb1 (Rb1, 12.5-100 $\mu \mathrm{M})$ on dye-coupling between cultured cells ( $\mathrm{n}=4$; $\mathrm{P}<0.05$, vs. control group). (D) The effects of 4-h cell treatment with ginsenoside $\operatorname{Rg} 1$ (12.5-100 $\mu \mathrm{M})$ or (E) notoginsenoside R1 $(12.5-100 \mu \mathrm{M})$ on connexin expression in HeLa cells ( $\mathrm{n}=4$; ${ }^{\mathrm{P}} \mathrm{P}<0.05$, vs. control group). GJ, gap junction; $\mathrm{Cx}$, connexin.

A

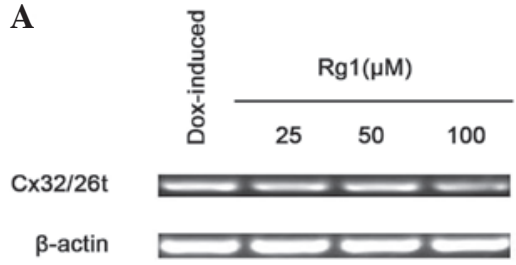

B

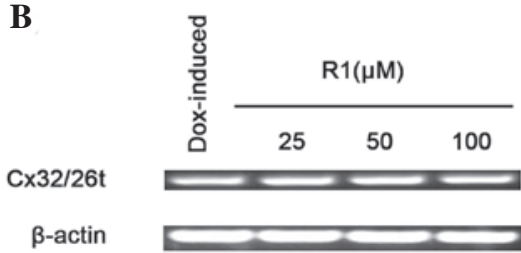

Figure 5. Effects of ginsenoside Rg1 and notoginsenoside R1 on Cx32/Cx26 mRNA levels. (A) Cells were treated with ginsenoside $\operatorname{Rg} 1(\operatorname{Rg} 1,25-100 \mu \mathrm{M})$ or (B) notoginsenoside R1 (R1, 25-100 $\mu \mathrm{M}$ ) for $4 \mathrm{~h}$, following which mRNA expression was assessed using RT-PCR. Agarose gel electrophoresis of RT-PCR products indicates no alteration of $\mathrm{Cx} 32 / \mathrm{Cx} 26 \mathrm{mRNA}$ levels following the treatments. Cx, connexin; RT-PCR, reverse transcription polymerase chain reaction.

have no effect if all cells responded identically. However, there is a range of sensitivities to toxic agents in cell populations. Therefore, specific cells are more sensitive and others are less, leading to more or less toxic signals in response. GJIC transmits a 'death signal' from sensitive to less sensitive cells, thus, enhancing the cytotoxicity of cisplatin in the entire cell population. In the current study, cells exposed to $0.5 \mu \mathrm{g} / \mathrm{ml}$ cisplatin at low-density conditions, without GJ, revealed a $17 \%$ cell death rate; however, with the formation of GJs, i.e. at high-density conditions, there was a cell death rate of $51 \%$. GJIC increases the cytotoxic activity of cisplatin by transmitting the "death signal' from sensitive to less sensitive cells. This indicates that elevating the intercellular spread of the 'death signal' by ginsenoside Rg1 and the notoginsenoside R1-induced enhancement of GJ formation or function may be responsible for the increased cytotoxic action of cisplatin. However, ginsenoside Rb1 had no effect on the cytotoxicity of cisplatin in the presence or absence of functional GJs, as observed in the results showing that ginsenoside Rb1 had no effect on dye-coupling and failed to transmit a 'death signal' from sensitive to less sensitive cells via GJs.

Although the propagation of the 'death signal' produced by stimulating pharmacological agents and radiation through GJIC has been widely investigated, the exact molecules responsible for this effect have not yet been identified (12,16-18). A number of possible signals are usually considered, including the toxic drug itself or its metabolites and molecules involved in the cellular death pathway. Cisplatin and its cytoplasmic aquated species have a molecular mass of $\sim 300 \mathrm{Da}$, which is much less than the upper limit of GJ-permeable molecules, indicating that these species may permeate GJs. Since cisplatin exerts its cytotoxic effects primarily by forming a variety of DNA adducts, intrastrand and interstrand cross-links (ICLs), Hong et al examined the effects of GJIC on cisplatin-induced formation of DNA ICLs at low and high cell densities (8). However, the results showed that GJIC had no effect on the 
formation of DNA ICLs. This result indicates that cisplatin and its immediate metabolites may not be the responsible toxic signals transferred among cells. The increase in cytotoxicity is hypothesized to be caused by the transfer of other toxic factors and further investigations are required to identify them.

In the present study, $100 \mu \mathrm{M}$ ginsenoside $\mathrm{Rg} 1$ and notoginsenoside R1 significantly increased the cytotoxicity of cisplatin via increased GJ formations. Thus, the dosage of cisplatin may be reduced, resulting in fewer side effects. PNS and its components may be developed as nontoxic chemoadjuvants that are used to increase the efficacy of anticancer chemotherapies by the upregulation or maintenance of GJ function. The current study also reveals a novel strategy in which the upregulation of GJs may be utilized to increase the efficacy of chemotherapy.

\section{Acknowledgements}

The present study was supported by grants from the China Postdoctoral Science Foundation (no. 20090461139), the Natural Science Foundation of the Provincial Education Department of Anhui (no. KJ2008A167) and the National Natural Science Foundation of China (no. 81001457).

\section{References}

1. Wang CZ, Luo X, Zhang B, Song WX, Ni M, Mehendale S, Xie JT, Aung HH, He TC and Yuan CS: Notoginseng enhances anti-cancer effect of 5-fluorouracil on human colorectal cancer cells. Cancer Chemother Pharmacol 60: 69-79, 2007.

2. Wang CZ, Xie JT, Zhang B, Ni M, Fishbein A, Aung HH, Mehendale SR, Du W, He TC and Yuan CS: Chemopreventive effects of Panax notoginseng and its major constituents on SW480 human colorectal cancer cells. Int J Oncol 31: 1149-1156, 2007.

3. Zhang CL, Yu ML, Tao L, Jiang Z and Liu H: Decreasing toxicity and synergistic effects of Panax notoginseng to tumor-bearing mice treated by cytoxan. Nanjing Zhongyiyao Daxue XueBao 4: 254-256, 2008 (In Chinese).

4. Krutovskikh VA, Piccoli C and Yamasaki H: Gap junction intercellular communication propagates cell death in cancerous cells. Oncogene 21: 1989-1999, 2002.
5. Lin JH, Weigel H, Cotrina ML, Liu S, Bueno E, Hansen AJ, Hansen TW, Goldman S and Nedergaard M: Gap-junction-mediated propagation and amplification of cell injury. Nat Neurosci 1: 494-500, 1998

6. Wang Q, You T, Yuan D, Han X, Hong X, He B, Wang L, Tong X, Tao L and Harris AL: Cisplatin and oxaliplatin inhibit gap junctional communication by direct action and by reduction of connexin expression, thereby counteracting cytotoxic efficacy. J Pharmacol Exp Ther 333: 903-911, 2010.

7. Yu ML, Zhang CL, Yuan DD, Tong XH and Tao L: Panax notoginseng saponins enhances the cytotoxicity of cisplatin via increasing gap junction intercellular communication. Biol Pharm Bull 35: 1230-1237, 2012.

8. Hong X, Wang Q, Yang Y, Zheng S, Tong X, Zhang S, Tao L and Harris AL: Gap junctions propagate opposite effects in normal and tumor testicular cells in response to cisplatin. Cancer Lett 317: 165-171, 2012.

9. He B, Tong X, Wang L, Wang Q, Ye H, Liu B, Hong X, Tao L and Harris AL: Tramadol and flurbiprofen depress the cytotoxicity of cisplatin via their effects on gap junctions. Clin Cancer Res 15: 5803-5810, 2009.

10. Koreen IV, Elsayed WA, Liu YJ and Harris AL: Tetracycline-regulated expression enables purification and functional analysis of recombinant connexin channels from mammalian cells. Biochem J 383: 111-119, 2004.

11. Goldberg GS, Bechberger JF and Naus CC: A pre-loading method of evaluating gap junctional communication by fluorescent dye transfer. Biotechniques 18: 490-497, 1995.

12. Jensen R and Glazer PM: Cell-interdependent cisplatin killing by $\mathrm{Ku} / \mathrm{DNA}$-dependent protein kinase signaling transduced through gap junctions. Proc Natl Acad Sci USA 101: 6134-6139, 2004.

13. Erdlenbruch B, Nier M, Kern M, Hiddemann W, Pekrun A, and Lakomek M: Pharmacokinetics of cisplatin and relation to nephrotoxicity in paediatric patients. Eur J Clin Pharmacol 57: 393-402, 2001.

14. Tao L and Harris AL: 2-aminoethoxydiphenyl borate directly inhibits channels composed of connexin 26 and/or connexin 32. Mol Pharmacol 71: 570-579, 2007.

15. Tanaka M and Grossman HB: Connexin 26 gene therapy of human bladder cancer: induction of growth suppression, apoptosis, and synergy with cisplatin. Hum Gene Ther 12: 2225-2236, 2001.

16. Mesnil $\mathrm{M}$ and Yamasaki H: Bystander effect in herpes simplex virus-thymidine kinase/ganciclovir cancer gene therapy: role of gap junctional intercellular communication. Cancer Res 60: 3989-3999, 2000.

17. Mesnil M, Piccoli C, Tiraby G, Willecke K and Yamasaki H: Bystander killing of cancer cells by herpes simplex virus thymidine kinase gene is mediated by connexins. Proc Natl Acad Sci USA 93: 1831-1835, 1996.

18. Azzam EI, de Toledo SM and Little JB: Oxidative metabolism, gap junctions and the ionizing radiation-induced bystander effect. Oncogene 22: 7050-7057, 2003. 